\title{
DIVERSITÉ FLORISTIQUE DU PARC NATIONAL DU HAUT ATLAS ORIENTAL ET DES MASSIFS AYACHI ET MAÂSKER (MAROC)
}

\author{
Mohammed Sghir TALEB ${ }^{1 *} \&$ Mohamed FENNANE ${ }^{2}$ \\ ${ }^{1}$ Centre National de la Recherche Forestière, BP. 763, Avenue Omar Ibn Al Khattab, Rabat-Agdal \\ ${ }^{2}$ Institut Scientifique, BP. 703, Avenue Ibn Battouta, Rabat-Agdal \\ *Autor correpondencia: taleb05@yahoo.com
}

Recibido el 24 de enero de 2008, aceptado para su publicación el 18 de agosto de 2008 Publicado "on line" en octubre de 2008

RÉSUMÉ. Diversité floristique du Parc National du Haut Atlas Oriental et des massifs Ayachi et Maâsker (Maroc). Etant donné la rareté des études sur le Parc National du Haut Atlas Oriental et les massifs Ayachi et Maâsker, les connaissances sur la diversité floristique de ces régions, très accidentées, étaient très insuffisantes et fragmentaires. Actuellement la flore vasculaire du Parc, jbels Ayachi et Maâsker est estimée respectivement à 227, 140 et 96 espèces; la flore rare, menacée et/ou endémique constitue une part importante. Le nombre d'endémiques dans le Parc (espèces et sous espèces) représente $6.8 \%$ de l'ensemble des endémiques marocaines. Ce nombre est très important dans l'Ayachi avec $7.5 \%$. Dans le Maâsker, le nombre d'endémiques est évalué à $3.9 \%$ de l'ensemble des endémiques marocaines. La flore rare (espèces et sous-espèces) est représentée par $3.6 \%$ de la flore totale du Parc, $4 \%$ de la flore totale du jbel Ayachi et $4 \%$ de la flore totale du jbel Maâsker. Par contre le taux de la flore très rare est faible, respectivement de l'ordre de $1.9 \%, 2 \%$ et $2.4 \%$.

Mots clés. Diversité floristique, Endémisme, Parc National du Haut Atlas Oriental, Maroc.

SUMMARY. Flora diversity of the National Park of the Eastern High Atlas and the massifs Ayachi and Mâasker (Morocco). Considering the rare studies on the Eastern High Atlas National Park, Ayachi and Mâasker mountains, the knowledge on the flora diversity of these regions were very insufficient. Their flora is estimated currently 227, 140 and 96 species. The rare flora, threatened and/or endemic constitutes an important part. The number of endemics in the Park (species and subspecies) represents $6.8 \%$ of the endemics of Morocco. This number is very important in Ayachi (7.5\%). In Maasker, the number of endemics is about 3.9\% of the endemic of Morocco. The rare flora (species and subspecies) in the Park, Ayachi and Maasker is represented respectively by $3.6 \%, 4 \%$, and $4 \%$. On the other hand the very rare flora is poor respectively $1.9 \%, 2 \%$ and $2.4 \%$.

Key words. Flora diversity, Endemism, National Park of Eastern High Atlas, Morocco.

\section{INTRODUCTION}

L'échantillonnage exhaustif (330 relevés) réalisé dans le Parc National du Haut Atlas Oriental et les massifs Ayachi et Maâsker (fig. 1) a permis de dresser une liste 


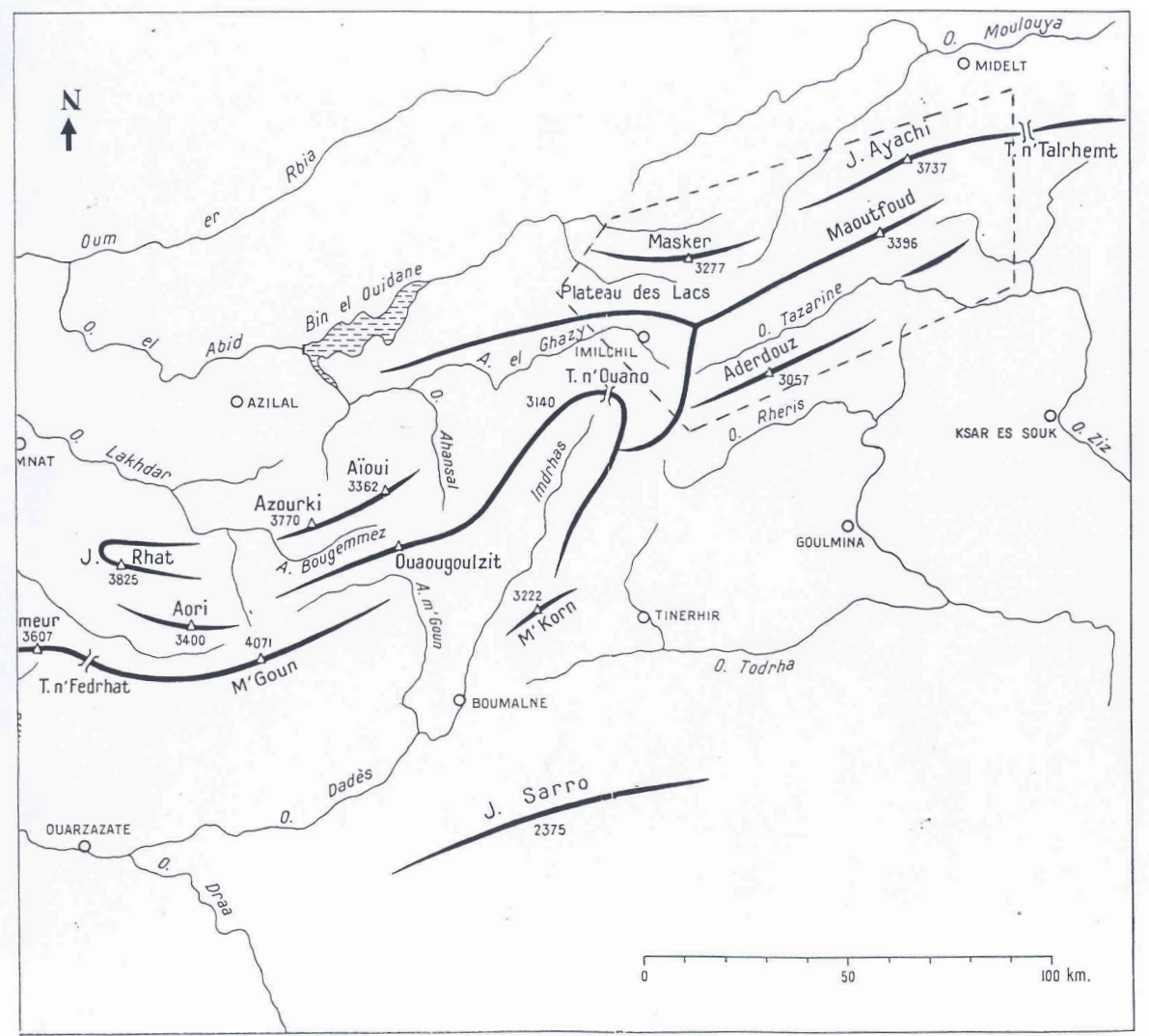

Figure 1. Carte schématique du Haut Atlas Oriental (Quézel, 1957); ----: Limite de la région étudiée.

floristique assez complète pour la première fois dans la région. Ce travail donne toutes les espèces échantillonnées ainsi que celles citées dans le Haut Atlas Oriental par le Catalogue des plantes du Maroc (Jahandiez \& Maire 1931, 1932 et 1934 ; Emberger \& Maire 1941). Les espèces signalées par Quézel (1957); Quézel \& al. (1987) ; Quézel $\&$ al. (1994) et Fennane \& Ibn Tattou (1998) ont été également ajoutées. Des précisions sur la chorologie de nombreuses espèces sont fournises en accordant une importance particulière aux espèces rares, menacées et/ ou endémiques.

\section{MATERIEL ET METHODE}

Ce travail concerne la diversité floristique du Parc National du Haut Atlas Oriental et ses bordures représentées par les massifs Ayachi et Maâsker.

Le Parc National du Haut Atlas Oriental couvre environ $49.000 \mathrm{Ha}$. Il s'étend sur deux provinces (Khénifra et Errachidia) et deux cercles (Midelt et Imilchil). Il occupe la frange altitudinale comprise entre 1.650 m (Oued N'Igli, Assif N-Oughaddou) et $3.077 \mathrm{~m}$ (Jbel Msedrid). Le domaine forestier représente environ 18.500 ha, soit $37 \%$ de 
la superficie totale du Parc. Les principaux affleurements géologiques sont représentés par des conglomérats calcaires datant du Dogger, du Bathonien ou du Bajocien; des marno-calcaires du Dogger, du Bathonien ou du Bajocien; des calcaires, calcaires dolomitiques ou calcaires marneux de l'Aalénien; des gabbros, basaltes et dolérites du Crétacé et des argiles rouges triasiques.

Jbel Ayachi, point culminant du Haut Atlas Oriental $(3.757 \mathrm{~m})$ domine la plaine de la Moulouya. C'est un massif complexe constitué d'une série de chaînons parallèles plus ou moins élevés protégeant la zone axiale. Sur le plan géologique, Jbel Ayachi est dominé par des calcaires marneux, graveleux d'âge Pleinsbachien avec un affleurement du calcaire dolomitique du Lias inférieur sur les hauts versants. La présence de nombreuses grandes falaises donne au jbel Ayachi un aspect très particulier.

Jbel Maâsker moins élevé (sommet à $3.265 \mathrm{~m}$ ) prolonge vers l'Ouest le massif de l'Ayachi au delà de la cluse de l'Oued Ansegmir. Sur le plan géomorphologique, il est caractérisé par l'affleurement de grandes falaises très raides, généralement inaccessibles surtout les hauts versants nord. Les substrats géologiques sont représentés par des calcaires dolomitiques du Lias inférieur sur les hauts sommets, des calcaires marneux graveleux (Pliensbachien), des calcaires marneux ou pélitiques (Toarcien) sur les versants sud et par des dolérites et basaltes stratifiées (Trias) et des conglomérats sur le versant nord dominant Tounfite.

Les bioclimats dominants varient entre le semi-aride et le subhumide avec des précipitations variant entre 200 et $400 \mathrm{~mm}$.

Etant donné la diversité floristique de la région, il était nécessaire de réaliser un échantillonnage fin sur les différentes zones du parc et de ses bordures. L'échantillonnage a été réalisé à l'aide de relevés floristiques en tenant compte de l'altitude, l'exposition, la pente et le substrat géologique. Ainsi environ 330 relevés floristiques ont été réalisés. Ces mêmes relevés ont été exploités pour une étude phytosociologique (Taleb, 2002).

Au laboratoire ainsi qu'à l'herbier national de l'Institut Scientifique (RAB), nous avons procédé à la détermination des espèces récoltées (un herbier a été concçu contenant nombre important d'échantillons d'espèces). Ensuite, nous avons établi une liste contenant les espèces notées et/ou récoltées sur le terrain ainsi que toutes les autres signalées dans le Haut Atlas Oriental par le Catalogue des plantes du Maroc (Jahandiez \& Maire 1931, 1932 et 1934 ; Emberger \& Maire 1941) et par Quézel (1957) ; Quézel et al-. (1987); Quézel et al. (1994). Par la suite, en se basant sur le Catalogue des plantes vasculaires rares, menacées ou endémiques du Maroc (Fennane \& Ibn Tattou, 1998), nous avons sélectionné les espèces rares, menacées ou endémiques présentes dans notre dition.

Pour les taxons inventoriés (espèce, sous-espèce ou variété) les informations fournies concernent: la rareté, l'endémisme au Maroc et dans la dition, le nom scientifique parfois avec synonyme (s) et la répartition géographique dans la dition et au Maroc. Les taxons échantillonnés ou notés sur le terrain sont marqués par un astérisque.

Pour la rareté et l'endémisme, nous nous sommes basés sur le catalogue des plantes vasculaires rares, menacées ou endémiques du Maroc (Fennane \& Ibn Tattou, 1998).

EM: Endémique du Maroc. V: Vulnérable au Maroc. R?: Soupçonné rare au Maroc. RR ?: Soupçonné très rare au Maroc. R: Rare au Maroc. RR: Très rare au Maroc

Afin de mettre l'accent sur l'endémisme strict dans le Haut Atlas et principalement dans la région étudiée, nous avons adopté aussi les catégories suivantes :

EHA: Endémique du Haut Atlas. EHAO: 
Endémique du Haut Atlas Oriental. EAy: Endémique du jbel Ayachi. EMaâsker: Endémique du jbel Maâsker. Eparc: Endémique du Parc.

Pour donner une idée sur l'abondance globale de chaque espèce échantillonnée dans la région étudiée, nous avons adopté les deux catégories suivantes: (a): taxon abondant à très abondant. (b): taxon peu abondant à rare.

Afin d'avoir une idée sur la répartition biogéographique des espèces au Maroc, des précisions sur la répartition des espèces dans les divisions géographiques ci-dessous ont été ajoutées.

AA: Anti Atlas. AS: Atlas Saharien. CN: Maroc central, partie septentrionale. CS: Maroc central, partie méridionale. ED: Maroc désertique oriental (de l'Algérie au Haut-Drâa). ES: Maroc oriental steppique (bassin de la Moulouya). HA: Haut Atlas (la présence ailleurs dans le Haut Atlas est indiquée par trois points de suspension sans détails). HAO: Haut Atlas Oriental. MA: Moyen Atlas. MAC: Moyen Atlas Central. MAO: Moyen Atlas Oriental. OL: Secteur oranais littoral (BeniSnassen). OS: Secteur oranais montagnard (montagnes de Debdou à Ghar-Rouban). R: Rif. S: Plaine du Sous. SW: Secteur macaronésien marocain (littoral et collines littorales du Cap Beddouza'à Ifni). T: Péninsule tingitane ou des Djebala, (limite Sud: Larache-Oued Laou). WN: Maroc occidental septentrional (de Larache à Casablanca). WS: Maroc occidental méridional (de Casablanca à Cap Beddouza). WT: Maroc désertique occidental (à l'W du Haut-Drâa). Z: Monts de Zaïan.

\section{RESULTATS}

PTERIDOPHYTA POLYPODIACEAE

Asplenium glandulosum Lois. $=A$. petrarchae (Guerin) DC.
HA (HAO...), R, AA, OL, WN.

Asplenium viride Huds.

HA (HAO...), MA.

Asplenium ruta-muraria L. var. subtenuifolium

Christ.

HA (Parc...), MA

Athyrium filix-femina (L.) Roth.

HA (HAO ...), R, MA, T, CN.

\section{SPERMATOPHYTA \\ CUPRESSACEAE}

*Juniperus communis L. subsp. hemisphaerica (C. Presl) Nyman (b)

RR. HA (Ayachi, Maâsker...), R (j. Tissouka; j. Lakraâ), MA (MAO:j. Bou-Naceur; j. Bou Iblane...).

*Juniperus oxycedrus L. (a)

HA (Ayachi, Maâsker, Parc...), R, MA

(MAC, MAO), AS, AA, Z, OL, OS, CN, CS.

*Juniperus phoenicea L. (a)

HA (Ayachi, Maâsker, Parc...), R, MA

(MAO...), AS, OS, T, WN, SW.

*Juniperus thurifera L. (a)

V.HA (Ayachi, Maâsker, Parc...), MA

(MAC, MAO), AA.

*Tetraclinis articulata (Vahl) Masters. (b)

HA (Parc...), R, MA (MAC, MAO), AA, OL, OS, T, CN, CS, WN, SW.

\section{PINACEAE}

*Cedrus atlantica Manetti (a)

HA (Ayachi, Maâsker, Parc...), R, MA

(MAC, MAO).

* Pinus halepensis Mill. (a)

HA (Ayachi, Maâsker, Parc...), R, MA

(MAC, MAO), AS, OS.

*Pinus pinaster Aiton $(\mathrm{b})$

HA (Ayachi, Maâsker, Parc...), R, MA

(MAC, MAO).

\section{TAXACEAE}

*Taxus baccata L. (b)

V.HA (Parc...), R, MA (MAC, MAO).

\section{EPHEDRACEAE}

*Ephedra major Host. (a)

= Ephedra nebrodensis Guss. MAO). 
SPERMATOPHYTA

(Angiospermae, Magnoliopsida)

\section{ACERACEAE}

*Acer monspessulanum L. (a)

HA (Maâsker, Parc...), R, MA (MAC, MAO), AS.

\section{ANACARDIACEAE}

*Pistacia atlantica Desf. (a)

HA (Parc...), R, MA (MAO...), AS, AA,

OL, OS, ED, ES, CN, CS, WN, WS, SW, H, S.

Pistacia terebinthus L. (b)

HA (HAO...), R, MA (MAC, MAO), AS, OL, OS, CN, WN, Z, CS.

\section{APOCYNACEAE}

* Nerium oleander L. (a)

HA (Parc...), R, MA, AA, OL, OS, ED, WD, ES, T, CN, CS, WN, Z, WS, SW, H, S.

\section{AQUIFOLIACEAE}

*Ilex aquifolium L. (a)

HA (Ayachi, Maâsker, Parc...), R, MA (MAC, MAO).

\section{ARALIACEAE}

*Hedera helix L. ssp. canariensis (Willd.) Maire (a)

HA (Parc...), R, MA (MAC, MAO), OL, OS, T, CN, WN.

\section{ASTERACEAE}

Achillea odorata L. var. microphylla (Willd.) Willk.

HA (Parc...), MA, AS.

Anacyclus atlanticus Litard. \& Maire var. vestitus Humbert

EM.HA (Ayachi...), MA (MAO...).

*Anacyclus pyrethrum (L.) Cass. var. depressus (Ball) Maire (b)

HA (Parc...), AS, AA.

Anacyclus valentinus $\mathrm{L}$

HA (Ayachi...), R, MA (MAO...), AA, OL, ES, T, SW, H.

* Anvillea radiata Cosson \& DR. var. genuina Maire (b)

HA (HAO ...), AA, ES, ED, WD, S.

*Artemisia alba Turra ssp. chitachensis Maire (b)
$=A$. chitachensis Cosson ex Batt. \& Trabut

EM.HA (Maâsker, Parc...), MA (MAO...).

*Artemisia herba alba Asso (a)

HA (HAO (Parc, Ayachi, Maâsker)...), R, MA (MAO...) , AS, AA, OS, ED, ES, T, H, SW,

$\mathrm{S}, \mathrm{OL}$.

*Artemisia mesatlantica Maire (a)

EM.HA (Ayachi, Parc...), MA.

*Artemisia negrei Ouyahya

EM.HA (Ayachi, Maâsker, Parc...), MA

(MAO...).

* Atractylis humilis L. ssp. eu-caespitosa (Desf.) Maire var. eu-caespitosa Maire (a) HA (HAO...), MA (MAO...), AS, OS, ES,

ED, H.

*Atractylis serratuloides Sieb. (a) HA (Ayachi, Parc...), WD, ES, ED.

*Bellis caerulescens Cosson (a)

EM.HA (Ayachi, Maâsker, Parc...), MA

(MAO...), AA.

Bellis silvestris Cyr. var. genuina Batt. HA (HAO...), R, MA, AA, OL, OS, T, CN, WN, Z, CS, WS, SW.

*Carduncellus atractyloides Batt. (a) R.HA (Ayachi, Maâsker, Parc...), MA

(MAO...).

*Carduncellus pomelianus Batt. (a) HA (Ayachi, Maâsker, Parc...), MA

(MAO...), CN.

* Carduncellus rhaponticoides Cosson \& Dur. (b) Hém.HA (Parc...), ES.

Carduus atlantis Humbert \& Maire subsp. megalatlanticus Emberger (b)

RR. EHA.HA (Ayachi...).

*Carduus ballii Hook EM.HA (Parc...), MA (MAC...), AA

Carduus chevallieri Berrate HA (Ayachi...), MA (MAO...), AS, AA.

* Carlina involucrata Poiret (b) HA (HAO...), R, MA, AS, AA, ES, T, WN, WS, SW, H.

Carthamus fruticosus Maire R ?. EM.HA (HAO...), AA.

*Catananche caerulea L. (a) HA (Ayachi, Maâsker, Parc...), R, MA (MAO...), AS, AA, OL, OS, CN.

*Catananche caespitosa Desf. (a) HA (Parc...), MA, AA.

* Centaurea benoistii Humbert (a) R ?. EM.HA (HAO...), MA (MAO...). 
Centaurea boissieri DC. subsp. atlantica (Font Quer) G. Blanca

EM.HA (HAO...), MA (MAC, MAO).

Centaurea gueryi Maire

R ?. EM.HA (Parc...), MA (MAC...).

*Centaurea incana Desf. (b) HA (Parc...), R, MA (MAO...), AS, AA,

OL, OS

*Centaurea josiae Humbert (b)

R. EHA.HA (Ayachi, Parc...).

Centaurea litardieri Jahandiez \& Maire var. villosa Maire

R. EM.HA (Ayachi...), MA (MAO...).

Centaurea musimonum Maire RR.HA (Parc...).

*Centaurea tenuifolia Dufour (a)

HA (Ayachi, Parc...), MA (MAC, MAO...),

$\mathrm{AA}, \mathrm{E}, \mathrm{ES}$.

Cirsium chrysacanthum (Ball) Jahandiez

R. EM. HA (Parc...), MA (MAO...), AA.

Cirsium dyris Jahandiez \& Maire var. genuinum Maire

AA.

EM.HA (Ayachi, Maâsker), MA (MAO...),

*Cirsium odontolepis Boiss. (b)

HA (Parc...), R, MA (MAC, MAO).

Crepis hookeriana Ball

R?. EM.HA (Ayachi...), R, MA (MAO...).

*Echinops spinosus L. ssp. bovei (Boiss.) Murbeck var. pallens Maire (a)

HA (Ayachi, Maâsker, Parc...), MA

(MAO...), AA, ED, WD, S, H.

Erigeron acer L. ssp. mesatlanticus Maire var. fuscomarginatus Emberger \& Maire HA (Ayachi, Parc...), ES.

*Hertia maroccana Maire (a) ED, ES

EM.HA (Ayachi, Parc...), MA (MAO...),

*Evax pygmaea (L.) Brot. (b)

HA (Maâsker, Parc...), R, MA, AA, OL, OS,

T, CN, WN, Z, CS, WS, SW.

*Hieracium amplexicaule L. (a)

R.HA (Ayachi, Maâsker, Parc...).

*Hieracium pseudopilosella Ten. (a)

HA (Ayachi, Maâsker, Parc...), R, MA

(MAC, MAO).

*Hypochoeris radicata L. (a)

HA (Maâsker...), R, MA, OL, OS, T, CN,

WN, Z, SW, WS, H, CS.

*Hypochoeris laevigata (L.) Ces. P. \& G. var. pinnatifida Doumergue (a)

HA (Ayachi...), R, MA, ES.

*Hypochoeris leontodoides Ball (a)

EM.HA (Ayachi...), MA (MAC...), AA.

*Inula viscosa (L.) Ait. var. typica Maire (a)

HA (Parc...), R, MA (MAC, MAO), AA,

OL, OS, ES, T, CN, WN, Z, CS, WS, SW, H, S.

*Inula montana L. (a)

HA (Parc...), R, MA (MAO...), AS, T.

*Jurinea humilis DC. (a)

HA (Parc...), MA (MAO...), AS, OS.

*Lactuca viminea (L.) Presl (a)

HA (Ayachi, Maâsker...), R, MA (MAO...),

AS, AA, T.

Lactuca reviersii Litard. \& Maire (a)

EM.HA (HAO...), MA (MAO...).

*Lactuca saligna L. (a)

HA (Ayachi, Maâsker, Parc...), R, MA

(MAO...), OL, OS, ES, SW, CN, WN, WS, H.

Lapsana communis L.

HA (Parc...), R, MA.

*Launaea acanthoclada Maire (a)

HA (Parc...), R, MA (MAO...), AS, AA,

ED, ES, H.

*Launaea arborescens (Batt.) Maire (a) HA (HAO ...), MA (MAO...), AS, AA, ES,

ED, H, SW, S, WD.

*Leontodon hispanicus Poiret ssp. euhispanicus Maire (b)

HA (Ayachi, Parc...), MA (MAO (Bou

Naceur)), R, AA, OS, ES.

*Leontodon pitardii Maire (b)

R?. EM. HA (Ayachi...), MA (MAO (Bou

Naceur)).

Leucanthemum atlanticum (Ball) Maire var.'euatlanticum Maire

$=$ Rhodanthemum atlanticum (Ball) Wilcox,

Bremer \& Humphries

R. EHA.HA (Ayachi...)

*Leucanthemum mairei Humbert (a) EM.HA (Ayachi, Maâsker, Parc...), R, MA

(MAO...).

Leucanthemum maresii (Cosson) Maire $=$ Rhodanthemum maresii (Cosson) Wilcox,

Bremer \& Humphries HA (HAO...), AS, OS.

Leucanthemum mesatlanticum Emberger \& Maire

$=$ Rhodantemum mesatlanticum (Emberger

\& Maire) Wilcox, Bremer \& Humphries 
RR. EM.HA (HAO...), MA (MAO...). Leucanthemum paludosum (Poiret) Bonnet \& Barr. ssp. decipiens (Pomel) Batt. var. gaetulum (Batt.) Maire HA (HAO...), AS, AA, ED.

Leucanthemum redieri Maire var. sericeum (Humbert) Maire

R. EM.HA (Ayachi...), MA (MAO...).

Leuzea berardioides Cosson

EM.HA (Ayachi...), R, MA (MAO...).

Leuzea conifera (L.) DC.

HA (HAO...), R, MA (MAO...), OS, T.

Mantisalca salmantica (L.) Briquet \& Cavill. HA (Parc...), R, MA, AS, AA, OL, OS, ES,

T, CN, WN, Z, CS, WS, SW, H.

*Micropus bombycinus Lag. (b) HA (Maâsker, Parc...), R, MA (MAO...), AS, AA, OL, OS, T, CN, Z, WN, SW, CS,S.

*Onopordon acaule L. (b) HA (Parc...), R, MA (MAO...), AS, OS.

*Ormenis scariosa (Ball) Litard. \& Maire $=$ Chamaemelum scariosa (Ball) Benedi (a) EM.HA (Ayachi, Parc...), MA (MAO...), AA.

*Ormenis africana (Jord. \& Fourr.) Litard. \& Maire (b)

HA (Parc...), MA, AS, ES.

* Phagnalon embergeri Humbert \& Maire (b) R. EM.HA (Ayachi...), MA (MAO...).

*Phagnalon helichrysoides (Ball) Cosson (a) EHA.HA (Parc...)

*Phagnalon rupestre (L.) DC. (a) HA (Parc...), R, MA, OL, OS, T, WN, CN, Z, CS, WS, SW, H.

* Scorzonera pygmaea Sibth. \& Sm. (a) HA (Ayachi, Maâsker, Parc...), R, MA (MAC, MAO), AA, OS.

Senecio lividus L. ssp. foeniculaceus (Ten.) Br.B1. \& Maire var. biennis Ball EAy.HA (Ayachi...).

Senecio chalureaui Humbert var.eradiatus Maire RR. EHA.HA (Ayachi...).

Serratula nudicaulis (L.) DC. var. subinervis Cosson HA (Maâsker...), MA.

*Sonchus maritimus L. var. eu-maritimus Maire (b)

HA (Parc...), R, MA, AS, AA, OL, OS, ES, ED, H, T, CN, WN, Z, CS,SW, SW, S, WD.

*Taraxacum atlantis-majoris Lindberg (b)
RR. EHA.HA (Parc...).

*Taraxacum humbertii Maire'(b)

RR. EHA.HA (Ayachi, Maâsker...).

Taraxacum microcephalum Pomel

HA (Ayachi...), MA, ES, H.

var. atlanticum Pomel

$=T$. atlanticum Pomel

RR. EM.HA (Ayachi...).

Tragopogon crocifolius $\mathrm{L}$.

HA, MA.

subsp. samaritana (Heldr. \& Sart. ex Boiss.) I.B.

K. Richardson

RR.HA (Parc...).

Tussilago farfara $\mathrm{L}$.

R.HA (Parc...), MA, ES.

*Xeranthemum inapertum (L.) Willd. (b)

HA (Parc...), R, MA (MAC, MAO), AS,

AA, OL, T, CN, Z, OS, CS.

* Berberis hispanica Boiss. \& Reut. (a)

HA (Ayachi, Maâsker, Parc...), R, MA

(MAC, MAO), AS.

\section{BORAGINACEAE}

*Echium velutinum De Coincy (b)

EM.HA (Parc...), AA, SW.

* Myosotis alpestris Schm., emend. Fiori ssp. typica Fiori (a)

HA (Ayachi, Maâsker, Parc...), MA (MAO

(Bou Iblane, Tichoukt, Bou Naceur, Guelb-erRahal), AA.

BUXACEAE

*Buxus balearica Willd. (a)

HA (Ayachi, Maâsker, Parc...), R, MA (MAO...), AS, OL, T.

\section{CAMPANULACEAE}

*Campanula filicaulis Dur. var. genuina Maire (a)

HA (Ayachi, Maâsker, Parc...), MA

(MAO...), AS, AA, CN.

Campanula guinochetii Quézel (b)

RR. EHA.HA (Ayachi...).

* Campanula mairei Pau ex Maire (a)

EHA.HA (Ayachi, Maâsker...).

*Jasione humilis Lois. (b)

$=J$. crispa (Pourret) Samp.

HA $($ HAO $($ Parc......), R, MA (MAO...),

OS.

*Legousia falcata Ten. var. scabra (Lowe) DC. 
(a)

HA $($ HAO $(\operatorname{Parc} \ldots) \ldots)$, R, MA (MAO...), OS, OL, T, CN, WN, WS, SW, Z.

\section{CAPPARACEAE}

*Capparis spinosa L. (a) HA (HAO (Parc.......), R, MA (MAO...), AA, OL, OS, ES, CN, SW.

var. aegyptiaca (Lam.) Boiss.

$=$ C. aegytiaca Lam.

HA (HAO (Parc...)...), MA (MAO...).

\section{CAPRIFOLIACE}

*Viburnum lantana L. (b)

HA (Parc...), MA.

*Lonicera arborea Boiss. (a)

HA (Ayachi, Maâsker, Parc...), R, MA

(MAO...).

*Lonicera etrusca Santi var. typicaHAlacsy (b) HA (Parc...), R, MA, T.

* Lonicera pyrenaica L. (a)

HA (Ayachi, Maâsker, Parc...), MA(MAO...).

\author{
CARYOPHYLLACEAE \\ * Arenaria armerina Bory (b) \\ RR.HA (Parc...), R, MA (MAO...).
}

* Arenaria dyris Humbert (a)

R. EHA.HA (Ayachi, Maâsker...).

* Arenaria pungens Lag. (a)

HA (Ayachi, Maâsker, Parc...), MA (MAO...).

*Arenaria serpyllifolia L. (a) HA (Ayachi, Parc...), R, MA, AS, AA, ES, T,

WN, WS, CN, CS, SW, Z, H.

*Buffonia murbeckii Emberger (a)

R. EHA.HA (Maâsker, Parc...).

*Cerastium gibraltaricum Boiss. var. boissieri (Gren.) Pau (a) HA (Parc...), R, MA (MAO...).

*Cerastium pumilum Curt. (a) HA (Parc...), MA.

*Corrigiola telephiifolia Pourret (b) HA (Ayachi, Parc...), R, MA, T, CN,WN,

WS, Z, CS, H.

Dianthus crinitus Sm. (b) HA (Parc...), MA (MAO...).

*Dianthus lusitanus Brot. (a) HA (Parc...), R, MA, AS, AA, ES, T, CN, WN, H, Z.

* Minuartia funkii (Jordan) Graebner (a) HA (HAO...), MA.
*Minuartia verna (L.) Hiern. (a)

HA (Ayachi...), MA (MAO...).

Paronychia aretioides DC. (b) RR.HA (Parc...).

* Paronychia argentea Lam. (a) HA (Parc...), R, MA (MAO...), ES, T, SW,

$\mathrm{H}, \mathrm{S}$.

var. velutina Ball

EHA.HA (Parc...).

*Paronychia capitata (L.) Lam. (a) HA (Ayachi, Parc...), R, OL, OS, ES, CN, WN, SW, H, S.

*Polycarpon polycarpoides (Biv.) Jahandiez \& Maire (a)

HA (Ayachi, Maâsker, Parc...), R, MA

(MAO...), AS, AA, OL, ES, OS.

* Silene ayachica Humbert (a) EM.HA (Ayachi...), MA (MAO...).

* Silene boryi Boiss. (a) HA (Ayachi, Parc...).

* Silene corrugata Ball (b)

EM.HA (HAO...), MA, AA, CN, CS, WS, SW, H, S.

* Silene cucubalis Wibel (a) $=S$. vulgaris $($ Moench) Garcke MAO).

HA (Maâsker, Parc...), R, MA (MAC,

*Silene dyris Maire (a)

R. EHA.HA (Ayachi...), MA (MAO...), AA.

Silene guinetii Quézel

RR. EHAO.HA (Ayachi, Maâsker, Parc...).

*Silene mesatlantica Maire (a)

EM.HA (Ayachi, Maâsker, Parc...), MA

(MAO...).

* Silene patula Desf. (b)

HA (Maâsker, Parc...), R, MA (MAO...),

AA, OL, OS, T, CN, Z, CS.

*Telephium imperati L. (a)

HA (Ayachi, Maâsker, Parc...), R, MA

(MAO...), AS, AA, OL, OS, ES, T, CN, CS.

\section{CHENOPODIACEAE}

* Fredolia aretioides (Bunge) Ulbr. (a)

$=$ Anabasis aretioides Bunge

HA (HAO...), AA, ED.

*Chenopodium vulvaria $\mathrm{L}$. (b)

HA (Parc...), R, MA, AA, OL, OS, ES, T,

CN, CS, Z, WN, WS, SW, H.

*Hammada scoparia (Pomel) Il'in (a) 
=Haloxylon scoparium Pomel

HA (HAO...), AS, AA, ES, WN.

*Noaea mucronata (Forssk.) Asch. \& Schw. (b) OL, ES. HA (HAO...), R, MA (MAO...), AS, OS,

Salsola vermiculata L. var. flavescens (Cav.) Moq. (a)

HA (Parc...), R, MA, AS, AA, OS, OL, ES, T, CN, WN, WS, SW, H.

\section{CISTACEAE}

* Cistus laurifolius L. (b)

HA (Parc...), R, MA (MAC...).

*Fumana ericoides (Cav.) Pau var. typica Pau (b)

HA (Ayachi...), R, SW.

*Fumana thymifolia (L.) Verlot (b) HA (Maâsker...), R, MA, AA, OL, OS, ES,

T, CN, CS, WN, WS, SW, Z.

*Helianthemum cinereum (Cav.) Pers. (a)

HA (Ayachi, Parc...), MA (MAO...).

*Helianthemum croceum (Desf.) Pers. (a)

= H glaucum (Cav.) Pers.

HA (Ayachi, Parc...), R, MA (MAC, MAO),

OL, OS, CN, Z, T.

*Helianthemum pergamaceum Pomel (a)

HA (Parc...), R, MA (MAO...), AS, AA, OL, OS, ES, ED, CN, CS, Z, H.

\section{CONVOLVULACEAE}

*Convolvulus arvensis $\mathrm{L}$. var. typicus Fiori (b) HA (Ayachi...), R, MA, AS, AA, OL, OS, ED, ES, T, CN, CS, WN, WS, SW, Z, H, S.

*Convolvulus mazicum Emberger \& Maire (a) EM.HA (Parc...), R, MA (MAC, MAO).

\section{CRASSULACEAE}

Sedum album L. ssp. gypsicolum (Boiss. \& Reut.) Maire var. glanduliferum Ball HA (HAO...), R, MA, AA, ES, OL, OS.

Sedum atlanticum (Ball) Maire

= Sedum surculosum Cosson

HA (HAO...), AA.

Sedum jaccardianum Maire \& Wilczek

R. EM. HA (Ayachi...), MA.

*Umbilicus horizontalis (Guss.) DC.

$=$ Cotyledon umbilicus-veneris DC. ssp. horizontalis (Guss.) Batt. (b) WS, H.

\section{CRUCIFERAE}

Alliaria officinalis DC.

$=$ Alliaria petiolata $(\mathrm{MB}$.) Cavara \& Grande HA (Parc...), R, MA (MAC...).

Alyssum alyssoides $\mathrm{L}$

R.HA (Maâsker...), R, MA (MAC...).

*Alyssum campestre L. var. typicum Maire (a) HA (Parc...), R, MA, AS, AA, OL, OS, ES, T, CN, CS, WN, WS, SW, H, S.

Alyssum cochleatum Cosson \& Dur. R.HA (Ayachi...), AS.

*Alyssum serpyllifolium Desf. (b) HA (Parc...), R, MA, AS.

*Alyssum spinosum L. (a) HA (Ayachi, Maâsker, Parc...), R, MA (MAC, MAO), AA.

Arabidopsis thaliana (L.) Schur. HA (HAO...), R, MA, CN.

* Arabis auriculata Lam. (a) HA (Parc...), R, MA (MAO...), AS, AA, OL, OS.

Arabis pubescens (Desf.) Poiret HA (Ayachi...), R, MA (MAO...), OL, OS,

T, CN, Z.

*Biscutella frutescens Cosson (a) HA (Maâsker, Parc...), MA, OS, T.

Brassica gravinae Ten. var. atlantica Batt. R ?. EHAO.HA (HAO...).

Brassica saxatilis (Lam.) Amo ssp. silenifolia Emberger

= Brassica repanda subsp. silenifolia

(Emberger) Greuter \& Burdet HA (Ayachi...)

* Draba hederefolia Cosson ssp. cossoniana (O. E. Schulz) Maire (a)

EM.HA (Ayachi, Maâsker...), MA (MAO...)

*Draba hispanica Boiss.; Maire (a) HA (Ayachi, Maâsker, Parc...), R, MA

(MAO...), AA.

Draba oreadum Maire ssp. mariae-aliciae (Emberger) Maire RR. Eay.HA (Ayachi...).

*Eruca sativa Lam. ssp. vesicaria (L.) Briq. HA (Parc...), ES, WS, SW.

*Erucastrum leucanthum Cosson \& Dur. var. genuinum Maire (a)

HA (Ayachi, Parc...), MA (MAO...), AS,

AA, ED, ES.

*Erysimum bocconei (All.) Pers. (a) 
= Erysimum nervosum Pomel

HA (Maâsker, Parc...), R, MA (MAO...), OL, OS, T.

*Erysimum incanum G. Kunze var. maroccanum (Pau \& Font Quer) Maire (a) = Erysimum incanum subsp. incanum HA (Parc...), R, MA (MAO...), AA.

*Iberis sempervirens L. (a) AS. HA (Ayachi, Maâsker...), MA (MAO...),

var. pseudosaxatilis (Maire) Greuter \& Burdet RR. EHA.HA (Maâsker, Rhate, Azourki, Imghal).

*Isatis tinctoria L. (a) HA (Parc...), MA (MAO...), AA.

* Matthiola scapifera Humbert var. genuina Litard. \& Maire (a)

EM. Hém.HA (Maâsker...).

*Moricandia arvensis (L.) DC. (b)

HA (Parc...), MA (MAC, MAO), AS, AA,

CN, ES, ED, WD.

*Raffenaldia platycarpa (Cosson) Stapf (b) $=$ Cossonia platycarpa Cosson

R. EHA.HA (Ayachi...).

* Raffenaldia primuloides Godron var. lutea Maire(b)

$=$ Cossonia africana Dur.

HA (HAO...), R, MA.

Sisymbrium maurum Maire

R. EM.HA (Parc...), MA.

*Vella mairei Humbert (a)

R. EM.HA (Ayachi, Parc...), MA (MAO...).

\section{DIPSACACEAE}

*Scabiosa parielii Maire var. typica Maire (a) HA (Ayachi, Parc...), AS, AA.

\section{EUPHORBIACEAE}

Euphorbia arvalis Boiss. \& Heldr.

R.HA (Ayachi, Parc...), MA.

*Euphorbia megalatlantica Ball (a)

EHA.HA (Ayachi, Maâsker, Parc...).

*Euphorbia nicaeensis All. (a) MAO).

HA (Ayachi, Maâsker, Parc...), MA (MAC,

\section{FAGACEAE}

*Quercus rodundifolia Lam.

= Quercus ilex L. (a)
HA (Ayachi, Maâsker, Parc...), R, MA

(MAC, MAO), AS, AA, OL, OS, T, CN, CS.

\section{GLOBULARIACEAE}

*Globularia alypum L. (a)

HA (Ayachi, Maâsker, Parc...), R, MA (MAO...), AS, AA, OL, OS, T, CN, Z, SW.

* Globularia liouvillei Jahandiez \& Maire (a)

R. EM.HA (Ayachi, Maâsker....), MA (MAO...).

*Globularia nainii Batt. (a)

EM.HA (Ayachi, Maâsker, Parc...), MA (MAO...).

\section{GUTTIFERAE}

Hypericum hyssopifolium var. candelabrum $\mathrm{Pau}$ \& Font Quer

RR.HA (Ayachi...), MA.

Hypericum tomentosum L. var. pubescens (Boiss.) Ball

HA (HAO ...), R, MA, OL, OS, T, CN, WN,

WS, CN, CS, Z, H, S.

subsp. wallianum Maire

RR. EHAO.HA (HAO).

\section{GERANIACEAE}

Erodium cheilanthifolium Boiss. ssp. cheilanthifolium Maire var. genuinum Maire

HA (Ayachi...).

*Erodium cicutarium (L.) L'Héritier (a) HA (Maâsker, Parc...), R, MA, OL, OS, ED,

ES, T.

*Geranium molle L. (a)

HA (Ayachi, Maâsker, Parc...), R, MA (MAC, MAO...) , AS, AA, OL, OS, ES, CN, CS, T, WN, WS, Z, SW, H.

*Geranium nanum Cosson (a) EM.HA (Ayachi...), MA (MAO...).

\section{LABIATAE}

* Ajuga iva (L.) Schreb. (a)

HA (Maâsker, Parc...), R, MA (MAO...), AA, OL, OS, ES, T, CN, WN, Z, CS, WS, SW, $\mathrm{H}, \mathrm{S}$.

* Dracocephalum renati Emberger (b)

R. EM. HA (Maâsker...), MA (MAO...).

*Lavandula multifida $\mathrm{L}$. (a)

HA (Parc...), R, MA (MAO...), AA, OL, OS, T, CN, WN, WS, SW, H, S, WD. 
* Lavandula tenuisecta Cosson

EM.HA (Parc...), AA.

Marrubium africanum (Litard. \& Maire) Humbert

= Marrubium litardierei Marmey

R. EM.HA (HAO...), AA.

*Marrubium ayardii Maire (a)

EM.HA (Ayachi, Parc...), MA (MAO...).

*Marrubium echinatum Ball (b)

EM.HA (Parc...), MA.

*Marrubium multibracteatum Humbert \& Maire (a)

EM.HA (Ayachi, Maâsker...), MA (MAO...)

Marrubium supinum L. (b)

HA (Maâsker...), MA (MAO...), AS.

*Marrubium vulgare $\mathrm{L}$. var. typicum Fiori (a) HA (Parc...), R, MA (MAO...), AS, AA,

OL, OS, ED, ES, T, CN, CS, Z, WN, WS, SW, H,

$\mathrm{S}$.

Mentha gattefossei Maire

EM. HA (Parc...), MA (MAC...), AA.

*Mentha longifolia (L.) Huds. (a)

HA (Parc...), MA (MAC...).

*Mentha pulegium L. (a)

HA (Parc...), R, MA (MAC, MAO), AS,

AA, OL, OS, ES, T, CN, CS, WN, Z, WS, SW, $\mathrm{H}$.

*Mentha rotundifolia L. ssp. rotundifolia Maire

(a)

HA (Parc...), R, MA (MAC, MAO), AS, AA,

OL, OS, ES, T, CN, CS, WN, Z, WS, SW, H.

Nepeta tuberosa $\mathrm{L}$.

HA (HAO...), R, MA, OS.

Nepeta barbara Maire

RR. EHAO.HA (Parc...).

Nepeta hispanica Boiss. \& Reut. ssp. statice Emberger \& Maire

R. EM.HA (Maâsker...), MA (MAC...).

* Nepeta nepetella L. ssp. amethystina (Poiret) Briq. var. atlantica Batt. (b)

HA (HAO...), MA (MAO...), AS, ES.

*Rosmarinus officinalis L. (a)

OS, OL

HA (Ayachi, Parc...), R, MA (MAO...), AS,

* Salvia aucheri Boiss. (b)

HA (Parc...), MA (MAO...).

* Salvia verbenaca $\mathrm{L}$. (a)

HA (Ayachi, Parc...), R, MA (MAO...), AA

OL, OS, ES, T, CN, CS, WN, Z, WS, SW, H, S,
WD.

*Satureja alpina (L.) Scheele (a)

HA (Maâsker, Parc...), , MA (MAO...).

* Satureja hochreutineri Briq. (b)

(Parc...), AS, AA, SW, H, S.

*Scutellaria orientalis L. ssp. demnatensis (Cosson) Batt. (a)

HA (Ayachi, Parc...), MA (MAO...).

*Sideritis incana L. (a)

HA (Ayachi, Parc...), R, MA (MAO...), AS.

* Sideritis montana L. var. ebracteata (Asso) Briq. in Hoch. (b)

HA (Parc...), R, MA (MAO...), AS, AA, OL, OS, ES, T, SW, H, S.

*Teucrium chamaedrys L. var. gracile Batt. (b) EM.HA (Parc...), R, MA (MAO...).

*Teucrium flavovirens Batt. (b) HA (Parc...), AS, ES, OS

*Teucrium mideltense (Batt.) Maire (a) EM.HA,HAO, ES.

*Teucrium musimonum Humbert var.genuinum Maire (a)

EM.HA (Ayachi, Maâsker, Parc...).

*Teucrium polium L. (a) HA (Parc...), R, MA (MAC, MAO...), AS,

AA, CN, T, SW, H, S.

*Teucrium rodundifolium Schreb. var. atlanticum (Ball) Maire (a) EM.HA (Ayachi, Parc...), MA.

*Thymus algeriensis Boiss. \& Reut. (b) HA (HAO...), MA.

* Thymus hirtus Willd. (b) $=$ Thymus willdenowii Boiss. HA (Parc...).

*Thymus pallidus Cosson (a) HA (Ayachi, Parc...), MA (MAO...), AA.

*Thymus satureioides Cosson (a) = Thymus commutatus (Batt.) Batt. EM.HA (Parc...), AA, SW.

* Thymus serpyllum L. ssp. ayachicus (Humbert) Maire (a) EHAO.HA (Ayachi, Maâsker, Parc...).

*Ziziphora hispanica L. (a) HA (Parc...), R, MA, AS, ES.

\section{LEGUMINOSAE}

*Adenocarpus bacquei Batt. \& Pitard (a) EM.HA (Parc...), MA, ES, ED.

*Anthyllis vulneraria L. (b) HA (Parc...), MA (MAC, MAO). 
*Astragalus armatus Willd. (a)

$=$ Astragalus armatus subsp. tragacanthoides Emberger

HA (Ayachi, Parc...), R, MA (MAO...), AS, AA, OS, ES.

*Astragalus boissieri Fisch. (a)

= Astracantha granatensis (Lam.) Podl. MAO).

HA (Ayachi, Maâsker, Parc...), MA (MAC,

Astragalus bourgaeanus Cosson

HA (Parc...), R, MA.

Astragalus froedinii Murbeck

RR. EHA. HA (HAO...).

*Astragalus ibrahimianus Maire (a)

EM. HA (Ayachi, Maâsker, Parc...), MA

(MAO...)

*Astragalus incanus L. (a)

HA (Ayachi, Maâsker, Parc...), R, MA

(MAO...), ES, CN.

Astragalus mairei Emberger \& Maire

= Astragalus maireanus Greuter \& Burdet

RR. EM.HA (Maâsker...), MA.

*Ceratonia siliqua L. (a)

HA (Parc...), R, MA (MAO...), AS, AA,

OL, OS, ES, T, CN, CS, Z, WN, WS, SW

*Cicer atlanticum Maire (b)

RR. EM.HA (Ayachi, Maâsker...), MA

(MAO...)

*Colutea arborescens L. (a)

$=$ Colutea atlantica Browicz

HA (Parc...), R, MA, OL, OS, T, SW, AS.

* Coronilla minima L. var. minima Maire (a) OS HA (Ayachi, Parc...), R, MA (MAO...), ES,

250. Coronilla scorpioides (L.) Koch HA (HAO...), R, MA (MAO...), AA, OL, OS, ES, T, CN, CS, Z, WN, WS, SW, H, S.

*Cytisus arboreus (Desf.) DC. (b) HA (Ayachi...), R, MA, OL, OS, T, CN, Z, WN, WS, SW.

*Cytisus balansae Ball (a)

HA (Ayachi, Maâsker, Parc...), R, MA

(MAC, MAO).

*Erinacea anthyllis Link (a)

HA (Ayachi, Maâsker, Parc...), MA (MAC, MAO), AS, AA.

*Genista scorpius DC. (a) HA (Parc...), MA (MAO...), AS, AA, ES.

*Hedysarum humile L.

= Hedysarum confertum Desf. (a)
ES, HA.

var. subspeciosum Emberger \& Maire

EM.HA (Ayachi, Parc...), MA (MAO...).

var. mesanthum Emberger \& Maire

EM.HA (Ayachi, Parc...), MA (MAO...).

var. fontanesii (Boiss.) Batt.

EM.HA (Parc...).

Hippocrepis liouvillei Maire

=Hippocrepis liouvillei subsp. genuina Emberger (b)

EM.HA (HAO...), MA (MAO...).

*Hippocrepis scabra DC.

= Hippocrepis monticola Lassen

HA (Ayachi, Parc...), R, MA (MAO...), AS, AA, ES.

var. grandiflora Emberger \& Maire

HA (Ayachi...), R, ES.

var. glauca Maire

HA (Ayachi...).

*Lotononis tapetiformis Emberger \& Maire (a)

RR. EHAO.HA (Ayachi, Maâsker, Parc...).

*Lotus maroccanus Ball (a)

EM.HA (Parc...), AA, ES, WN, CS, Z, CN,

WS, SW, H.

*Medicago suffruticosa Ramond ssp. maroccana (Batt.) Maire (b)

$=$ ?subsp. liocarpa (Benth.) Urban

EM.HA (Maâsker, Parc...), R, MA.

*Onobrychis argentea Boiss. ssp. africana (Sirj.) Maire var. eu-africana Maire (a) HA (Parc...), ES.

Onobrychis pallasii (Willd.) M. Bieb. var. ayachica Emberger \& Maire Eay.HA (Ayachi...).

Ononis antiquorum L.

= Ononis spinosa subsp. antiquorum (L.) Arcangeli

HA (Ayachi...), R, AA, WN,H, S.

*Ononis atlantica Ball (a)

R. EM.HA (Ayachi...).

*Ononis cenisia L.

$=$ Ononis cristata Miller

HA (Ayachi, Parc...), R, MA.

Ononis laxiflora Desf. ssp. eu-laxiflora Maire HA (Parc...), MA, AA, OL, CN, CS, WN, SW, H, S.

Ononis natrix L. (a)

HA (Ayachi, Parc...), R, MA (MAO...), AA,

OL, T, CN, WN, WS, H, S.

*Psoralea bituminosa L. var. typica Fiori (a) = 
Bituminaria bituminosa (L.) Stirton HA (Parc...), R, MA, AS, AA, OL, OS, T, $\mathrm{CN}, \mathrm{SW}, \mathrm{H}$.

* Retama sphaerocarpa (L.) Boiss. (a) HA (Parc...), MA, AS, ES, ED.

Tetragonolobus siliquosus (L.) Roth var. bicolor Maire

EM.HA (Maâsker...), ES

*Trifolium campestre Schreb. var. hionanthum (Hausskn.) Maly (b)

HA (Parc...), R, MA, OL, OS, ES, T, CN,

CS, Z, WN, WS, SW, H.

*Trifolium humile Ball (a)

EM.HA (Ayachi...), MA.

Trifolium pratense L. ssp. spontaneum Willk. HA (Ayachi...).

*Vicia glauca Presl var. ayachica Emberger (a) EHAO.HA (Ayachi, Parc...).

\section{LINACEAE}

Linum austriacum L. ssp. collinum (Guss.) Asch. var. mauritanicum (Pomel) Maire HA (HAO...), MA, OL, OS, ES, T.

*Linum suffruticosum L. (a) SW HA (Parc...), R, MA (MAO...), AS, ES, T,

\section{MALVACEAE}

*Malope malacoides L. ssp. tripartita (Boiss. \& Reut.) Maire (a)

HA (Ayachi, Parc...), MA, AA.

* Malva silvestris L. ssp. eu-silvestris Maire var. typica Fiori (a)

HA (Parc...), R, MA (MAO...), OL, OS, ES,

T, CN, CS, WN, WS, Z, SW, H, S.

OLEACEAE

*Fraxinus angustifolia Vahl var. australis (Gay) Maire (a)

HA (Parc...), R, MA (MAC, MAO), OL,

OS, T, CN, WN, WS, H.

*Fraxinus dimorpha Cosson \& Dur. $=$ Fraxinus xanthoxyloides Wall.

(a) HA (Ayachi, Maâsker, Parc...), MA (MAC, MAO), AS, AA.

\section{OROBANCHACEAE}

Orobanche aegyptiaca Pers. var. tacassea (Beck) Maire

HA (HAO ...)
PAPAVERACEAE (incl. Fumariaceae)

Fumaria segetalis (Ham.) Coutinho

$=$ Fumaria algeriensis Pugsley

RR.HA (Région de Tounfite...), MA

(MAC...), AS.

*Fumaria vaillantii Lois. (b) ssp. eu-vaillantii Maire var. typica Emberger \& Maire HA (Parc...).

ssp. schrammii (Valen.) Maire

= Fumaria pugsleyana (Pugsley)'Liden

var.'pugsleyana Maire

EM.HA (Parc...), MA (MAC, MAO...).

*Papaver dubium L. (b)

$=$ Papaver malviflorum Doumergue

HA (Parc...), R, ES, T, CN, WN, SW, H.

Platycapnos saxicola Willk.

HA (Ayachi...), MA (MAO...)

* Sarcocapnos crassifolia (Desf.) DC. (b)

HA (Parc...), MA.

\section{PLANTAGINACEAE}

*Plantago albicans L. (b)

HA (HAO...), R, MA (MAO...), AA, OL, OS, ED, ES, CN, CS, WS, SW, H, S.

*Plantago coronopus L. (a)

HA (Ayachi, Maâsker, Parc...), R, MA

(MAC, MAO...), AA, OL, OS, T, CN, WN, WS, $\mathrm{H}, \mathrm{S}, \mathrm{Z}$.

*Plantago mauritanica Boiss. \& Reut. var. maroccana Batt. (a)

EM. HA (Parc...), MA.

\section{PLUMBAGINACEAE}

* Statice plantaginea All. ssp. choulettiana (Pomel) Maire

=Armeria choulettiana Pomel

HA (Parc...), MA.

\section{POLYGALACEAE}

*Polygala rupestris Pourret var. saxatilis (Desf.) Murbeck (b)

$=$ Polygala saxatilis Desf.

HA (Maâsker...), R, MA, AA, ED, ES.

\section{POLYGONACEAE}

*Rumex atlanticus Batt. (b)

R. EM. HA (Ayachi, Parc...), R.

PRIMULACEAE

*Anagallis arvensis L. (b) 
HA (Parc...), R, MA (MAO...), AS, AA, OL, OS, T, CN, CS, Z, WN, WS, SW, H, S.

Coris monspeliensis L. var. maroccana Murbeck

EM.HA (Ayachi...), R, T, WN, WS, SW.

\section{RANUNCULACEAE}

Aconitum lycoctonum L. ssp. neapolitanum (Ten.) Litard. \& Maire var. rerayense Litard. \& Maire

EHA.HA (Maâsker...).

*Delphinium balansae Boiss. \& Reut. (b) R, MA (MAC...), AS, AA.

subvar. caeruleum Litard. \& Maire

EHA.HA (Ayachi...).

Ranunculus calandrinioides Oliver

EM.HA (HAO...), MA (MAC...), AA.

Ranunculus geraniifolius Pourret ssp. aurasiacus (Pomel) Maire var. mesatlanticus Maire

EM.HA (Ayachi...), MA (MAO...).

Thalictrum minus L. ssp. pubescens (Schleich.) Rouy \& Fouc. var. eu-pubescens Maire HA (Ayachi...), MA (MAC...).

\section{RESEDACEAE}

Reseda alba $\mathrm{L}$.

HA (Parc...), MA, AA, CN, CS, WN, WS,

SW, H, S.

Reseda luteola L. ssp. biaui (Pitard) Maire

HA (HAO...), R, MA (MAC...), AA.

* Reseda nainii Maire (b)

EM.HA (Parc...), MA, AA, ES.

Rhamnus alpina L.

RHAMNACEAE

R.HA (HAO ...), MA (MAO...).

*Rhamnus atlantica Murbeck (a)

EM.HA (Ayachi, Maâsker, Parc...), MA.

* Rhamnus pumila Turr. (b)

HA (HAO (Ayachi...)), R, MA (MAO...).

\section{ROSACEAE}

*Amelanchier ovalis Medik (a)

R.HA (Ayachi, Maâsker, Parc...), MA (MAC, MAO).

307*. Cotoneaster nummularia Fisch. \& Meyer (a)

HA (Ayachi, Maâsker, Parc...), R, MA

(MAC, MAO...).

308*. Crataegus laciniata Ucria (a)
= Crataegus orientalis subsp. preslia Christensen

HA (Ayachi, Maâsker, Parc...), R, MA (MAC, MAO), OS.

309* Potentilla guilliermondii Emberger \& Maire (b)

RR. EHA.HA (Ayachi, Parc...).

310*. Prunus mahaleb L. (b)

HA (Parc (Rocher calcaire du versant Sud du jbel Aberdouz; vers 2400 m)...), R, MA.

*Prunus prostrata Labill. (a)

HA (Ayachi, Maâsker, Parc...), R, MA

(MAC, MAO), AS, AA.

* Rosa canina L. (a)

HA (Parc...), R, MA, AA, CN, Z, OS, WN, $\mathrm{H}$.

* Rosa micrantha Sm. \& Sow var. diminuta (Bor.) Braun; Maire (b)

HA (HAO (entre Agoudim et Tagoudit)).

* Rosa obtusifolia Desv. var. obtusifolia (Desv.) Crépin (a)

HA (Maâsker, Parc...).

* Rosa sicula Tratt. (a)

HA (Ayachi, Maâsker, Parc...), R, MA

(MAC, MAO).

* Rubus ulmifolius Schott. (a)

HA (Parc...), R, MA (MAC, MAO), AA, OL, OS, ES, T, CN, CS, Z, WN, WS, SW, H, S.

* Sanguisorba minor Scop. (a)

HA (Ayachi, Parc...), R, MA (MAC, MAO),

AS, AA, ES, T, C, W, SW, H, S.

*Sorbus aria (L.) Crantz (a) HA (Maâsker, Parc...), R, MA (MAC...).

*Sorbus cf. hybrida L. (b)

RR.HA (Maâsker...).

\section{RUBIACEAE}

*Asperula cynanchica L. (a) ssp. aristata (L. f.) $\mathrm{B}, \mathrm{g}$.

HA, MA, OS, T, CN, SW.

var. breviflora Batt.

HA (Ayachi...).

*. Asperula hirsuta Desf. var. longifolia Maire (a)

HA (Parc...), R, MA (MAO...), AS, AA, OL, OS, ES, T, CN, WN, Z, CS, WS, SW, H.

Asperula litardierei Humbert

RR. EM.HA (Ayachi...), MA (MAO...).

* Callipeltis cucullaria (L.) Stev. (b) HA (Parc...), R, MA (MAO...), AS, AA, 
OS, OL, ES, ED, WD, S.

Galium fruticosum Willd. ssp. ephedroides Willk.

HA (HAO...), R, MA, AA, OL, OS, WD, ED.

*Galium mollugo L. (a) HA (Parc...), R, MA, CN .

*Galium pumilum Murr. (b) R.HA (Parc...), MA (MAO...)

*Rubia peregrina L. var. intermedia G. G. (b) HA (Ayachi...), R, MA, OL, OS, T, CN, CS, WN, WS, Z, SW, H.

\section{RUTACEAE}

Aplophyllum linifolium (L.) A. Juss.

= Haplophyllum linifolium (L.) G. Don fil. R?.HA (HAO...), MA, OS, ES.

Ruta montana $\mathrm{L}$.

HA (HAO...), R, MA, AA, OL, CN, SW, H,

S.

\section{SALICACEAE}

*Populus alba L. (a)

HA (Parc...), R, MA (MAC, MAO), AS, AA, OL, OS, ES, T, CN, CS, WN, WS, SW, H, S.

*Populus nigra L. (a)

HA (Parc...), R, MA, AA, CN.

* Salix alba L. (a) HA (Parc...), R, MA, T, CN, WN.

* Salix purpurea L. (a)

HA (Parc...), R, MA, AA.

\section{SAXIFRAGACEAE}

Ribes alpinum $\mathrm{L}$.

RR.HA (Ayachi...), MA (MAO...).

*Ribes uva-crispa L. (a) (Ayachi, Maâsker, Parc...), R, MA (MAC, MAO), AA.

*Saxifraga longifolia Lapeyr. (a)

RR.HA (Ayachi, Maâsker...), MA

(MAO...)

* Saxifraga pedemontana All var. demnatensis (Cosson) Emberger (a)

R?. EM. HA (HAO (Ayachi...)), MA (MAO...).

\section{SCROPHULARIACEAE}

*Anarrhinum fruticosum Desf. ssp. eufruticosum Maire (a) CN.
*Antirrhinum ramosissimum Cosson \& Dur. (a) HA (Parc...), AS, AA, ES, ED, WD, H, S.

*Digitalis lutea L. (a) R.

ssp. transiens (Maire) Emberger \& Maire var. dyris Maire

R. EHA. HA (Parc...).

ssp. cedretorum Emberger

RR. EHAO. HA (Maâsker...).

*Erinus alpinus L. (a)

R?. HA (Ayachi, Parc...), R.

*Linaria heterophylla Desf. (a) HA (Maâsker, Parc...), R, T, WN.

* Linaria munbyana Boiss. \& Reut. (b) RR. HA (Parc...), CN, WN, SW.

Linaria origanifolia (L.) DC. ssp. flexuosa (Desf.) Maire HA (Ayachi...), AS, OS.

* Linaria simplex (Willd.) DC. (a) HA (Parc...), R, MA (MAO...), AS, AA, OL, OS, T, CN, Z, WN.

*Linaria tristis (L.) Mill. ssp. lurida (Ball) Maire var. genuina Maire (a)

R ?. EM.HA (Ayachi, Maâsker...).

Linaria ventricosa Cosson EM. HA (Parc...), AA, SW, H, S.

* Scrophularia canina L. var. typica Fiori (a) HA (Parc...), R, MA, AS, AA, OL, OS, ES,

T, CN, WN, WS, SW, H, S, WD.

* Scrophularia ramosissima Lois. ssp. macrorrhyncha Humbert, Litard. \& Maire (a) = Scrophularia macrorrhyncha (Humbert,

Litard. \& Maire) Ibn Tattou

RR. EM. HA (Ayachi, Maâsker, Parc...), MA (MAO...).

*Verbascum hookerianum Ball var. ballii Murbeck (a)

EM. HA (Parc...), AA.

*Verbascum lychnitis L. (a) R. HA (Parc...), R, MA.

var. giganteum Maire EM. HA (Parc...), R, MA.

*Veronica rosea Desf. (a) var. atlantica Ball HA (Maâsker, Ayachi...), R, MA, AS, AA. var. glabrescens Maire HA (Parc...).

\section{SOLANACEAE}

Withania adpressa (Cosson) Batt. HA (HAO...), AA, WD, ED. 
THYMELAEACEAE

*Daphne laureola L. var. latifolia Cosson (a) HA (Maâsker...), R, MA (MAC...).

*Thymelaea virescens Cosson \& Dur. (a) HA (Parc...), MA, AS, OS.

* Thymelaea virgata (Desf.) Endl. (a) OS, T. HA (Parc...), R, MA (MAO...), CN, H, Z

\section{UMBELLIFERAE}

Balansaea glaberrima (Desf.) Maire HA (HAO...), R, MA, OL.

Bupleurum album Maire

R. EM.HA (Ayachi...), AA.

*Bupleurum atlanticum Murbeck (a) HA (Ayachi, Maâsker, Parc...), MA (MAO...)

Bupleurum benoistii Litard. \& Maire EM.HA (Ayachi...), MA (MAC...).

Bupleurum lateriflorum Cosson EM.HA (Parc...), AA.

*Bupleurum spinosum L var. genuinum Maire (a)

HA (Ayachi, Maâsker, Parc...), R, MA (MAC, MAO), AA, OS.

*Carum atlanticum Litard. \& Maire (b)

R. EHA.HA (Ayachi...).

Carum lacuum Emberger (b)

RR. Eparc.HA (HAO (Parc...)...).

Caucalis daucoides $\mathrm{L}$.

$=$ Caucalis platycarpos $\mathrm{L}$.

R.HA (Parc...), R, MA.

* Caucalis leptophylla L. (b)

HA (Parc...), R, MA, AA, OL, OS, CN, Z, CS, WN, WS, SW, H.

*Eryngium bourgati Gouan var. hispanicum Lange (a)

HA (Ayachi, Maâsker, Parc...), MA (MAO...).

*Heracleum sphondylium L. ssp. montanum (Sch.) Briq. (a)

HA (Parc...), MA, AA.

Laserpitium emilianum Emberger

R. EHAO.HAO (Parc...)

Petroselinum hortense Hoffmg. var. gracillimum Maire

HA (Ayachi...)

* Pimpinella tragium Vill. (a) HA (Ayachi, Parc...), MA (MAO...), AS

*Pituranthos scoparius (Cosson \& Dur.) Benth. \& Hook. var. eu-scoparius Maire (a)
HA (HAO...), R, MA (MAO...), AS, AA, ES, ED, WD, H.

Scandicium stellatum (Soland.) Thell. var. decipiens (Bornm.) Thell.

HA (Parc...).

*Seseli libanotis (L.) Koch. ssp. atlanticum Maire (a)

EM.HA (Ayachi...).

*Seseli nanum (L.) Duf. (a)

HA (Ayachi, Parc...), MA.

*Torilis nodosa (L.) Gaertn. (a)

HA (Parc...), R, MA, AS, AA, OL, OS, ES,

ED, T, CN, CS, WN, WS, SW, H, S, WD.

\section{VALERIANACEAE}

*Centranthus angustifolius DC.

$=$ Centranthus lecoquii Jordan (a)

R.HA (Ayachi, Parc...), R, MA (MAO...),

OL.

*Centranthus calcitrapa (L.) Duf. (b)

HA (Parc...), R, MA, AA, OL, OS, T, CN, CS, WN, WS, Z, SW, H, S.

\section{VIOLACEAE}

Viola dyris Maire var. orientalis Emberger

R?. EHA.HA (Ayachi...).

*Viola maroccana Maire (a)

R?. EM.HA (Parc...), MA (MAC...).

*Viola saxifraga Maire (a)

EM.HA (Ayachi, Parc...), MA (MAC, $\mathrm{MAO})$.

\section{ZYGOPHYLLACEAE}

Fagonia malvana Maire \& Weiller

RR. EHAO.HA (Ayachi...).

*Peganum harmala L. (a)

HA (HAO...), R, MA (MAO...), AS, AA,

WD, ED, ES, CN, CS, WS, SW, H, S.

\section{SPERMATOPHYTA}

(Angiospermae, Liliopsida) ALLIACEAE

*Allium margaritaceum Sm. var. robostum Maire (b)

HA (Parc (Plateau des lacs)...).

\section{ASPARAGACEAE}

* Asparagus stipularis Forsk. (b)

HA (HAO...), R, MA, AA, OL, ED, ES, T, CN, CS, WN, WS, Z, SW. 


\section{ASPHODELACEAE}

Asphodelus ayardii Jahandiez \& Maire

R. EM. HA (Ayachi...), MA.

Asphodelus fistulosus L. var. atlanticus Jahandiez, Maire \& Weiller (b)

EM. HA (Parc...), AA.

*Asphodelus microcarpus Viv. var. eu-microcarpus Maire (b)

HA (Parc...), R, MA (MAO...), AS, AA, ES,

T, CN, CS, WN, WS, Z, SW, H, S.

\section{Carex hordeistichos Vill.}

HA (Parc (Lac Tislit)...), MA.

Crocus nudiflorus Sm.

\section{IRIDACEAE}

= Crocus multifidus Ram.

RR. HA (Ayachi...), R, MA.

\section{JUNCACEAE}

Juncus articulatus L. var. genuinus (P. Cout.) Briq.

HA (Parc (Lac Tislit)...), MA, T, SW.

Juncus compressus Jacq. ssp. gerardi (Lois.) Rouy WD.

R?.HA (Parc (Lac Tislit)...), MA (MAC ...),

Juncus fontanesii J. Gay ssp. brachyanthus Trab. var. melanocephalus Trab.

EM.HA (HAO...), MA, AA, ED.

Juncus punctorius L. var. mauritanicus (Trab.) Buchen. \& Trab.

HA (HAO...), MA, AS.

Triglochin palustre $\mathrm{L}$.

HA (Parc (Pelouses entre Imilchil et lac Tislit)...), R, MA.

\section{ORCHIDACEAE}

Platanthera algeriensis Batt. \& Trab.

R?. HA (Ayachi...), MA.

\section{POACEAE}

*Aegilops ovata L. ssp. eu-ovata Eig. var. vulgaris Cosson \& Dur. em. Eig. (a) HA (Parc...), R, MA (MAC, MAO), AS, AA, OL, OS, ES, T, CN, CS, Z, WN, WS, H, S. Agropyrum cristatum (L.) Gaertn.

R. HA (Parc (Plateau des lacs)...), MA,
Agropyrum festucoides Maire =Elymus festucoides (Maire) Ibn Tattou EHA.HA (Maâsker, Parc...).

Agropyrum marginatum Lindberg var. typicum Maire \& Weiller

HA (Parc...), MA (MAC...).

Agropyrum pseudofestucoides Emberger \& Maire = Elymus festucoides (Maire) Ibn Tattou

EHAO.HAO.

var. muticum Emberger HA (Parc (Lac Tislit)...).

var. acutiflorum Emberger HA (Ayachi...).

Agropyrum repens (L.) P. Beauv. var. arvense Rchb.

HA (Ayachi (Oued Tarart)...) R, MA, OL, OS, ES, T, CN, CS, Z, WN, WS, SW, H.

*Agrostis alba L. ssp. eu-alba Litard. (a) HA (Parc...), R, MA, OL, OS, ES, T, CN, WN, WS, SW, CS, H.

*Agrostis verticillata Vill. (a) HA (Parc...), R, MA, AS, AA, OL, OS, ES, ED, T, CN, CS, WN, WS, SW, H, S.

Alopecurus pratensis $\mathrm{L}$. ssp. ventricosus (Cosson) Thell. var. liouvilleanus (Br.-B1.) Maire HA (Parc (Lac Tislit)...), MA, OS.

*Arrhenatherum elatius (L.) Mert. \& Koch. ssp. erianthum (Boiss. \& Reut.) Trab. (a)

HA (Maâsker, Parc...), R, MA (MAC...) OL, OS, T, CN, WN, WS.

*Avena bromoides Gouan (a) HA (Parc...), R, MA (MAC, MAO), AS, AA, OL, OS, T, CN, Z.

Avena filifolia Lag. HA (HAO...), MA (MAO...), AS.

*Avena jahandiezii Litard. (a) EM.HA (HAO ...), MA (MAC, MAO).

410*. Avena montana Vill. (a) HA (Ayachi, Maâsker, Parc...), MA (MAO...).

*Avena sterilis L. ssp. macrocarpa (Moench) Briq. (a)

HA (Parc...), R, MA (MAO...), AS, AA, OL, OS, ES, T, CN, CS, WN, WS, SW, H, S.

*Brachypodium distachyum (L.) Roem. \& Schult. (b)

HA (Parc...), R, MA (MAO...), AS, AA, OL, OS, ES, T, CN, CS, WN, WS, SW, H, S.

*Brachypodium phoenicoides (L.) Roem. \& 
Schult. (b)

HA (Parc...), R, MA, T, CN, WN, Z.

*Bromus erectus Huds. (a) ssp. eu-erectus Asch. $\&$ Gr.

HA (Ayachi...).

ssp. permixtus Lindberg var. embergeri Maire HA (Maâsker...)

*Bromus squarrosus L. (a)

R, MA, HA, AS.

var. typicus Posp. AA.

HA (Parc...), R, MA (MAC, MAO), AS,

*Bromus tectorum L. (a)

HA (Ayachi, Maâsker, Parc...), R, MA

(MAO...), AS, AA, OL, OS, T, CN, Z.

*Cynodon dactylon (L.) Pers. (a)

HA, R, MA, AS, AA, OL, OS, ES, ED, T, $\mathrm{CN}, \mathrm{CS}, \mathrm{WN}, \mathrm{WS}, \mathrm{SW}, \mathrm{WD}$.

var. genuinum Maire

HA (Parc...), R, MA (MAC, MAO), AS, AA, OL, OS, ES, ED, T, CN, CS, WN, WS, SW, WD.

*Cynosurus elegans Desf. ssp. obliquatus (Link) Trab. (a)

HA (Parc...), R, MA (MAC, MAO), OL, T, CN, Z.

*Dactylis glomerata L. (a)

HA (Ayachi, Maâsker, Parc...), R, MA

(MAC, MAO), AS, AA, ES, T, Z, CN, WN, WS

SW, H, S.

*Echinaria capitata (L.) Desf. (b) HA (Maâsker...), R, MA (MAC, MAO), AS

AA, OL, OS, ES, CN, CS, H.

Eremopyrum bonaepartis (Sprengel) Nevski

RR. HA (Parc (Plateau des lacs)...), ES.

*Festuca deserti Cosson \& Dur. ssp. maroccana (Trab.) St.-Yves (a)

RR. EM?.HA (HAO...).

*Festuca elatior L (a)

HA (Ayachi, Parc...), R, MA (MAO...), ES,

T, C, W, SW, H, S.

Festuca fontqueri St.-Yves

EM.HA (Ayachi, Maâsker, Parc...), R, MA, AA.

*Festuca geniculata L. ssp. eu-geniculata Maire (a)

HA (Parc...), R, MA (MAC, MAO), AS, AA, ES, T, CN, CS, WN, WS, SW, H, S, Z.

*Festuca hystrix Boiss. (a) HA (Ayachi, Parc...), MA (MAO...), T.
*Festuca mairei St.-Yves (a) HA (Ayachi, Parc...), AS, OS.

*Festuca ovina L. ssp. indigestaHAck. var. maâskerensis Litard. (a)

= Festuca maâskerensis (Lit.) Romo

RR. EMaâsker ..HA (Maâsker).

Festuca rubra L. ssp. nevadensisHAck. var. gaetula Maire

HA (HAO...).

Festuca scaberrima Lange $\mathrm{HA}, \mathrm{HAO}, \mathrm{R}, \mathrm{MA}$.

Festuca triflora Desf.

WN, Z.

HA (Ayachi...), R, MA, AS, OS, CN, CS,

Haynaldia hordeacea (Cosson \& Dur.)HAck. HA (Parc...), R, MA (MAC...), AS, AA.

*Hordeum murinum L. ssp. leporinum (Link.) Asch. \& Graebn. (a)

HA (Ayachi, Parc...), R, MA (MAC, MAO), AS, AA, OL, OS, ES, ED, T, CN, CS, Z, WN, WS, H, S, WD.

Koeleria caroli Emberger EHA.HA (HAO...).

*Koeleria splendens Presl (a)

R. HA (Ayachi, Maâsker, Parc...), R, MA

(MAO...).

Koeleria vallesiana Honckeny var. typica Domin. HA (Parc...), MA, AS, ES.

*Lygeum spartum $\mathrm{L}$. (a) HA (HAO...), R, MA, AS, ED, ES, CN, CS, $\mathrm{H}, \mathrm{S}$.

* Melica cupani Guss. (a)

HA (Ayachi, Maâsker, Parc...), R, MA (MAC, MAO), AA, SW.

*Oryzopsis caerulescens (Desf.) Richter (a) HA (Ayachi, Parc...), R, MA (MAO...), AS, AA, OL, OS, T, WN, SW, H.

Oryzopsis paradoxa (L.) Nutt. HA (Ayachi, Parc...), R, MA, T.

* Phragmites communis Trin. var. isiacus (Del.) Cosson \& Dur. (a) HA (Parc...), R, MA (MAC, MAO), AS, T,

CN, CS, WN, WS, SW, H, S, Z.

*Poa alpina L. (b) HA (Parc...), MA

* Poa annua L. (a) HA (Parc...), R, MA, AA, OL, OS, ES, T, $\mathrm{CN}, \mathrm{CS}, \mathrm{Z}, \mathrm{WN}, \mathrm{WS}, \mathrm{SW}, \mathrm{H}, \mathrm{S}$.

*Poa bulbosa L. (a) HA (Ayachi...), R, MA (MAC, MAO), AS, 
AA, ,OL, OS, ES, T, CN, CS, Z, WN, WS, SW, $\mathrm{H}, \mathrm{S}$.

* Stipa barbata Desf. (a)

HA (Maâsker, Parc...), R, MA (MAO...),

AS, AA, OL, OS, ES, Z.

Stipa fontanesii Parl.

= Stipa balansae H. Scholz.

R ?.HA (Ayachi...), R, MA (MAC...), OL, OS.

* Stipa lagascae Roem. \& Schult. (a)

HA (Parc...), R, MA, AS, OS.

*Stipa nitens Ball (a)

EM.HA (Ayachi, Maâsker, Parc...), MA

(MAO...), AA.

* Stipa parviflora Desf. (a)

HA (Ayachi, Parc...), R, MA (MAO...), AS, AA, OL, ED, ES, H, SW.

* Stipa pennata L. ssp. mediterranea (Trin. \& Rupr.) Asch. \& Gr. (b)

HA (Parc...), MA (MAO...).

* Stipa tenacissima L. (a)

HA (Ayachi, Parc...), R, MA (MAO...), AS,

AA, OL, OS, ES, SW.

*Trisetum flavescens (L.) P. Beauv. (a)

HA (Maâsker, Parc...), R, MA (MAC, MAO), AA, OS, T, CN, WN.

POTAMOGETONACEAE

Potamogeton pectinatus $\mathrm{L}$. $\mathrm{H}, \mathrm{S}$.

HA (Parc (Lac Tislit)...), MA, CN, WN, SW,

ZANNICHELLIACEAE

Zannichellia palustris $\mathrm{L}$.

HA, MA, ES, T, CN, WN, WS, H

ssp. pedunculata (Reichb.) Murbeck

= Zannichellia pedunculata Reichb.

RR.HA Parc (Lac Tislit)...).

\section{DISCUSSION}

\section{Diversité floristique du Parc National du Haut} Atlas Oriental

L'analyse quantitative et qualitative de la flore du Parc a permis d'inventorier 227 espèces, 21 sous espèces et 55 variétés réparties entre 56 familles et 192 genres.
La flore rare et menacée à l'échelle du Maroc et présente dans la dition compte une seule espèce soupçonnée rare, 11 espèces rares et 5 espèces très rares. Nous signalons également la présence d'une seule sous-espèce soupçonnée rare: Juncus compressus ssp. gerardi et une seule très rare: Zannichelia palustris ssp. pedunculata.

La flore endémique du Parc est remarquable par sa richesse. Elle compte environ 46 espèces, 7 sous-espèces et 12 variétés. Parmi les espèces endémiques, 8 sont rares $(\mathrm{R}), 6$ très rares $(\mathrm{RR})$ et 2 soupçonnées rare ( $\mathrm{R}$ ?).

Les sous espèces et variétés endémiques constituent une part importante du nombre total des endémiques marocaines rencontrées dans le Parc. Elles représentent 41\% dont $15 \%$ de sous-espèces et $26 \%$ de variétés.

La flore endémique rencontrée dans le Parc appartient à 17 familles. L'analyse de sa répartition en fonction des familles montre que les Astéracées sont les plus riches avec 14 taxons (13 espèces et 1 sous-espèce). La famille des Labiées est aussi riche avec environ 11 taxons ( 7 espèces, 1 sous-espèce et 3 variétés). Les Légumineuses comptent 8 taxons (3 espèces, 1 sous-espèce et 4 variétés), les Scrophulariacées 4 taxons (2 espèces, 1 sous-espèces et 1 variété) ; les Graminées 3 espèces et 1 variété; les Caryophyllacées 3 espèces et 1 variété ; les Ombellifères et les Crucifères 3 espèces chacune; les Violacées 2 espèces ; les Convolvulacées, les Polygonacées, les Rhamnacées, les Résédacées et les Rosacées sont faiblement représentées et ne contiennent qu'une seule espèce chacune. Une seule sousespèce endémique représente les Papavéracées dans le Parc. Enfin, les Plantaginacées et les Liliacées ne sont représentées que par une seule variété chacune.

\section{Diversité floristique du jbel Ayachi}

Le massif de l'Ayachi abrite aussi une 
flore relativement riche et variée, comptant 197 taxons (140 espèces, 15 sous-espèces et 42 variétés). Cette flore s'organise en 42 familles.

Le diagnostic de la flore rare et menacée met en évidence 10 taxons ( 8 espèces, 1 sous espèce et 1 variété) rares, 3 espèces pour la catégorie soupçonnée rare et 2 espèces très rares.

La diversité orographique du jbel Ayachi et son altitude élevée ont permis l'installation et l'individualisation d'une flore très riche en taxons endémiques, soit environ 72 taxons dont 49 espèces, 6 sousespèces et 17 variétés. Parmi les espèces endémiques 3 espèces sont soupçonnées rares, 14 espèces sont rares et 8 espèces sont très rares.

Les sous espèces et variétés endémiques constituent une part importante de l'ensemble des endémiques rencontrées dans le jbel Ayachi, on en compte 6 sous-espèces et 17 variétés (soit environ $40 \%$ du nombre total des endémiques) dont 3 sous-espèces très rares, 2 variétés rares, 2 variétés très rares et une variété soupçonnée rare.

Les taxons endémiques se répartissent entre 23 familles. Les Asteracées sont les plus importantes avec 23 taxons (13 espèces, 2 sous-espèces et 8 variétés), ce qui représente environ $1 / 3$ du nombre total de taxons endémiques. Les Légumineuses, les Caryophyllacées et les Labiées contiennent respectivement 9 , 6 et 5 taxons. Les Crucifères et les Ombellifères ont 4 taxons endémiques chacune ; les Graminées ont 3 taxons, les Globulariacées, les Géraniacées, les Ranunculacées et les Violacées contiennent 2 taxons chacune. Enfin les autres familles (Crassulacées, Euphorbiacées, Polygalacées, Primulacées, Rhamnacées, Rosacées, Rubiacées, Saxifragacées, Asphodelacées, Scrophulariacées et Zygophyllacées) ne montrent pas plus d'un seul taxon chacune.

Le nombre d'endémiques du jbel Ayachi est très faible: trois taxons seulement ( 2 sous espèces et une variété) dont une sous espèce très rare.

La flore vulnérable est représentée par le genévrier thurifère qui organise des formations généralement dégradées et parfois même très dégradées où cette espèce n'est représentée que par des pieds morts.

\section{Diversité floristique du jbel Maâsker}

Le jbel Maâsker constitue un pôle biogéographique important où s'individualise une flore relativement pauvre mais remarquable par sa richesse en endémiques et en plantes saxicoles. Cette flore compte environ 96 espèces, 9 sous-espèces et 18 variétés qui se répartissent en 31 familles.

La richesse spécifique confirme la nette dominance des Astéracées et des Graminées avec successivement $16(16.6 \%)$ et 11 espèces $(11.4 \%)$. Cette richesse est relativement faible chez les Légumineuses et les Caryophyllacées avec $8(8.3 \%)$ espèces chacune, les Crucifères et les Rosacées avec $6(6.2 \%)$ espèces chacune. Les Labiées contiennent 5 (5.2\%) espèces, les Cupressacées, les Pinacées et les Globulariacées ont $3(3.1 \%)$. Les Caprifoliacées, les Géraniacées et les Euphorbiacées contiennent 2 (2\%). Enfin, les autres familles (Gnétacées, Acéracées, Aquifoliacées, Berbéridacées, Boraginacées, Buxacées, Campanulacées, Cistacées, Fagacées, Oléacées, Plantaginacées, Rhamnacées, Scrophulariacées et Ombellifères) ne contiennent qu'une seule $(1 \%)$ espèce chacune.

La flore endémique du jbel Maâsker représente environ $31 \%$ de la flore totale du massif. Elle compte 25 espèces, 6 sous espèces et 7 variétés qui se répartissent entre 12 familles et 21 genres.

Les sous espèces ou variétés endémiques constituent aussi une part importante de la flore endémique du jbel Maâsker. Elles représentent environ $34 \%$ du nombre total des endémiques. 
La répartition des taxons endémiques par famille montre que les Asteracées, les Légumineuses et les Labiées sont les plus riches en taxons endémiques avec 6 taxons chacune. Les Caryophyllacées et les Graminées contiennent 5 taxons chacune. Les Crucifères contiennent 3 taxons. Les Euphorbiacées et les Globulariacées montrent 3 taxons chacune. Viennent ensuite les Campanulacées, les Ranunculacées, les Rhamnacées et les Scrophulariacées avec un seul taxon endémique.

Il convient aussi de signaler que contrairement au jbel Ayachi (4 endémiques strictes) et au Parc (1 endémique stricte), jbel Maâsker n'abrite aucune endémique stricte.

\section{BIBLIOGRAPHIE}

EMBERGER L. \& R. MAIRE -1941 - Catalogue des plantes du Maroc. Tome IV. Complément aux volumes I, II et III. Minerva, Alger.

FENNANE M. \& M. IBN TATTOU -1998Catalogue des plantes vasculaires rares, menacées ou endémiques du Maroc. Bocconea $8.243 \mathrm{p}$.

FENNANE M. \& M. S. TALEB -2000- Sorbus L. Brève description et commentaire. Acta
Bot. Malacitana $25: 256$.

JAHANDIEZ E. \& R. MAIRE -1931-Catalogue des plantes du Maroc (Spermatophytes et Ptéridophytes). Tome I. Ptéridophytes, Gymnospermes et Monocotylédones. Minerva, Alger.

JAHANDIEZ E. \& R. MAIRE -1932- Catalogue des plantes du Maroc Spermatophytes et Ptéridophytes). Tome II. Dicotylédones, Archichlamydées. Minerva, Alger.

JAHANDIEZ E. \& R. MAIRE -1934-Catalogue des plantes du Maroc Spermatophytes et Ptéridophytes). Tome III. Dicotylédones Gamopétalées et supplement aux volumes I et II. Minerva, Alger.

QUEZEL P. -1957- Peuplements végétaux desHAutes montagnes de l'Afrique du Nord. Essai de synthèse biogéographique et phytosociologique. Edit. Lechevalier Paris, $463 p$.

QUEZEL P., M. BARBERO \& A. BENABID 1987- Contribution à l'étude des groupements forestiers et pré-forestiers du Haut Atlas oriental (Maroc). Ecol. Méditerraner. XIII $(1,2)$ : 107-117.

QUEZEL P., M. BARBERO, A. BENABID \& S. RIVAS-MARTINEZ -1994- Le passage de la végétation méditerranéenne à la végétation saharienne sur le revers méridional du Haut Atlas oriental (Maroc). Phytocoenologia 22 (4): $537-582$. 